\title{
Modification of Earth-Space Rain Attenuation Model for Earth- Space Link
}

\author{
Assadeq Abolhaoshat Mansour Albendag ${ }^{1}$, Ahmad FaizalMohd. Zain ${ }^{2}$ \\ ${ }^{1,2}$ Faculty of Science and Technology, UniversitiSains Islam Malaysia, Malaysia
}

\begin{abstract}
Rain attenuation is the primary source of signal degradation especially for those operating at frequencies up to $10 \mathrm{GHz}$. Achieving precise prediction of the rain attenuation to certain location is quite difficult due to the inhomogeneity of the rain along the propagation path. Vertical and horizontal reduction factors playing a significant role to estimate the effective path length precisely by reducing the actual path length penetrated in the rain. This paper presents the rain attenuation prediction based on the knowledge of rain microstructure parameters such as the raindrop size, rainfall rate and the raindrops size distribution. The drop size distribution is calculated based on Marshall-Palmer model. The total link attenuation has been calculated using the modified model proposed in [1] to find the total effective path attenuation of HAPS earthspace link located in Kuantan Malaysia $\left(3.80^{\circ} \mathrm{N}, 103.32^{\circ} \mathrm{E}\right)$. The frequency of the predicted signal has been carried out in this study is $31 \mathrm{GHz}$, while the polarization which used is circular polarization.
\end{abstract}

Keywords: HAPS; rain attenuation; rain prediction mode; Ka-band fade; rain cell size attenuation.

\section{Introduction}

The demands to providing a developed wireless communication infrastructure, to ensure high-quality communication services to the customers, has led to develop a new communications technology. The terrestrial and satellite are part of the communication infrastructure where they are used to providing a mobile communications services. It is based on quasi-stationary platforms operating in the stratosphere able to provide the wireless communications via High Altitude Platform Station (HAPS). HAPS is a new leading innovation dedicated for delivering wireless communication services from Stratosphere layer to customers on ground. It can be manned or unmanned aerial platforms located at altitude between 17-25 km above the Earth's surface.

Rain attenuation is the primary source of signal impairment for HAPS communication systems operating at any frequency above $10 \mathrm{GHz}$. The attenuation due to raindrops is greater than the attenuation due to other forms of precipitation. Rain attenuation is caused by absorption and scattering of the signal, in which the raindrop acts as poor dielectric. The drop of rain decays the power of radio waves and dissipates it by scattering and absorption. Raindrops cause greater attenuation by scattering than absorption at frequencies above $100 \mathrm{MHz}$ $[2,3]$. Scattering is an interaction process between the path of the electromagnetic waves and rain particles. Due to the mechanism of the high signals is scattering, thus each particle scatters energy from the incident signal and reradiates that energy to all directions, causing not only forward scatter, but also side and back scatters as shown in figure. The particle is then will be like an optical gap to the incident wave. The scattering occurs only when the refractive index of the particle be different from the air medium, thus the scattering takes place whenever an electromagnetic wave propagates through different medium. In the atmosphere, the scattering of the higher microwave signals is that the size of raindrops is close to the wavelength.

Gaseous molecules exists in the atmosphere absorb energy from radio waves passing through them, thus causing attenuation. This attenuation is greatest for some molecules such as water $\left(\mathrm{H}_{2} \mathrm{O}\right)$ and oxygen $\left(\mathrm{O}_{2}\right)$. Each type of absorbing molecule contributes a general background level of absorption, which are proportional linearly with the frequency. Figure (1) illustrates the normal atmospheric conditions which only $\mathrm{O}_{2}$ and $\mathrm{H}_{2} \mathrm{O}$ contribute significantly to absorption, although other atmospheric gases may be significant in very dry air at above $70 \mathrm{GHz}$. The main resonance peaks of $\mathrm{O}_{2}$ are at 60 and $118.74 \mathrm{GHz}$, whereas those of $\mathrm{H}_{2} \mathrm{O}$ are 22.3, 183.3 and $323.8 \mathrm{GHz}$. [4]. 


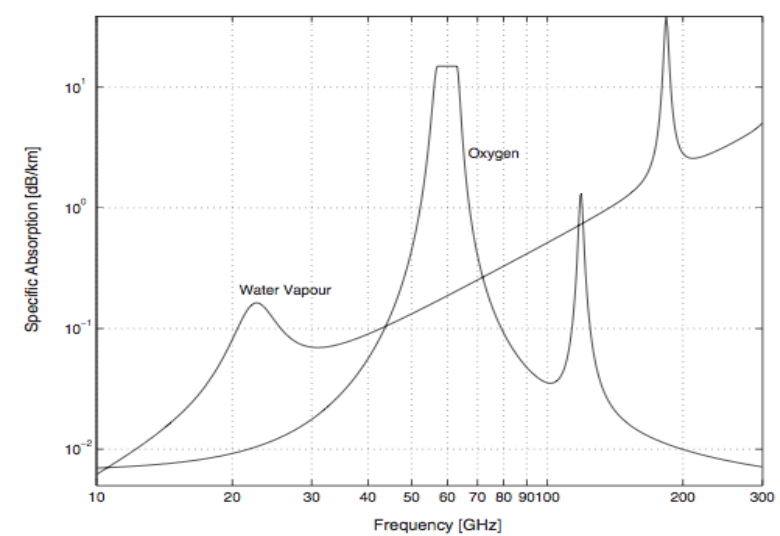

Figure 1. Specific attenuation of water vapor and oxygen. [ITU-P676-9, 12]

The aim of the attenuation modeling is to provide a suitable method for communication system designers to predict the amount of the attenuation for the communication links. Many models are in use in different regions in the world to estimate the impact of rain on the signal links. The prediction efficiency is varies among those models based on the signal link characteristics as well as the geographical characteristics of the location of interest.

\section{Methodology}

In millimeter-wave range of the radio spectrum the number and the size of the rain particles play a significant role for signal degradation, because the scattering and absorption due to rain is dependent on the drop size distribution. DSD can be interpreted as the number of raindrops per unit volume. These parameters have to be well understood to develop and/or evaluate the precipitation prediction models.

The attenuation of rain based on the knowledge of rain microstructure requires such model that specifies the rain rate and the size distribution of the raindrops.

\section{A. Raindrop Size Distribution (DSD)}

The raindrop distribution is denoted by $\mathrm{N}_{(\mathrm{D})}$ with units of $\mathrm{m}^{-4}$, DSD provide one of the most comprehensive representation to the natural raindrop distributions [5], where D is the drop radius. Rain formalized by drops of many different diameters, thus the actual drop size distribution might be vary widely in rain storms, but the average distribution is almost stable [6]. The DSD for different rain drops size and different rainfall intensities will be examined in this chapter.

\section{B. Marshall-Palmer DSD}

The raindrop size distribution has been studied by many investigators and generally modeled as an exponential distribution. For the tropical regions, the most widely DSD used in scientific literature is Marshall and Palmer model [7], which is an exponential distribution with two fitting parameters $\mathrm{N}_{0}$ and $\Lambda \square$. A brief description of Marshall-Palmer DSD can be described by follows

$$
\mathrm{N}_{(\mathrm{D})} \square \square \mathrm{N}_{0} \mathrm{e}^{-\Lambda \mathrm{D}} \square \square \square \square \square \square \square \square \square \square \square\left[\mathrm{m}^{-4}\right]
$$

WhereN $_{(D)}$ is the number of rain drops per cubic meter $\mathrm{m}^{-3}, \mathrm{~N}_{0} \square 8 * 10^{6}$, D is the drop size diameter in unit of meters and $\Lambda$ and $\mathrm{N}_{0} \square$ are empirical constants determined from the measured distributions. $\Lambda$ is related to rain rate $\mathrm{R}(\mathrm{mm} / \mathrm{hr})$ as follow

$$
\Lambda \square \square 4100 \mathrm{R}^{0.21}\left[\mathrm{~m}^{-1}\right]
$$

Rain rate $\mathrm{R}$ is a measure of the intensity of rain by calculating the volume of rain that falls on the ground in a certain time. The rain rate is expressed in units of length (depth) per unit time ( $\mathrm{mm} / \mathrm{hr}$ ), which is the depth of rain captured by a collection vessel per unit time. 


\section{Rain Cross Section}

The rain attenuation A of a radio wave propagating in a volume of rain of extent $\mathrm{d}$ in the direction of wave propagation can be modeled by integrating the cross-section of rain drop over complete signal path penetrated in rain and it can be expressed as

$$
A=\int_{0}^{d} \gamma d x \quad[d B]
$$

From the fundamentals of electromagnetic theory, it has been established that magnitude of an electromagnetic wave traveling through a rain-filled medium in a distance $\mathrm{d}$ decays in the amplitude by a factor of $\mathrm{e}^{-\gamma \mathrm{d}}$ where $\gamma \square$ is the attenuation coefficient [dB/km] [8] [9], which is given by

$$
\gamma=\mathrm{N}_{0} \mathrm{Q}_{\mathrm{t}}
$$

where $\mathrm{N}_{0}$ is the number of rain drops per unit volume, and $Q_{t}$ is the attenuation cross-section due to the drop of radius a. The attenuation cross section is a summation of the absorption $Q_{a}$ and scattering $Q_{s}$ for each drop.

The principle of signal attenuation of radio waves in $\mathrm{dB}$ can be written as

$$
A_{d B}=10 \log \frac{1}{e^{-\gamma d}}(5)
$$

wheree $=2.718$, therefore the signal attenuation can be written as

$$
\mathrm{A}_{\mathrm{dB}}=4.343 \gamma \mathrm{d}
$$

Where $\gamma$ is the specific attenuation and it can be written as:

$$
\gamma=Q_{t}(r, \lambda, m) N_{(D)}
$$

In the real situations, the raindrops are not all have a uniform radius, therefore the attenuation coefficient must be determined by integrating over all of the drop sizes.

$$
\gamma=4.343 \int \mathrm{Q}_{\mathrm{t}}(\mathrm{r}, \lambda, \mathrm{m}) \mathrm{N}_{(\mathrm{D})} \mathrm{dr}
$$

The above equation shows the dependence of rain attenuation on drop sizes, drop size distribution, rain rate, as well as the drop cross section.

Raindrops cross-section $Q_{\mathrm{t}}$ is found by applying the Mie scattering theory for a plane wave radiated through an absorbing sphere. [10]

$$
Q_{t}=\frac{\lambda^{2}}{2 \pi} \sum_{n=1}^{\infty}(2 n+1) \operatorname{Re}\left[a_{n}+b_{n}\right](9)
$$

where $a_{n}$ and $b_{n}$ are Mie scattering coefficients.

Using (1) and (9), the rain specific attenuation in $\mathrm{dB} / \mathrm{km}$ is expressed by

$$
\gamma=4.343 \int_{0}^{\infty} \mathrm{Q}_{\mathrm{t}}(\mathrm{r}, \lambda, \mathrm{m}) \mathrm{N}_{(\mathrm{D})} \mathrm{dr}(10)
$$

where $Q_{t}$ is the attenuation cross section of rain drop and it can be obtained from (9). The integral in (10) can be calculated numerically for specified values of and drop size distribution, refractive index and frequency.

\section{Analysis And Discussion}

Rain rate is the volume of water that falls to the surface of the earth in the form of droplets measured by unit area per unit time $[\mathrm{mm} / \mathrm{hr}]$. Rain precipitation is the primary source of the electromagnetic energy depletion during the propagation in rainy medium, caused by raindrop absorption. The specific attenuation obtained from (10) as a function in rainfall rate for different frequencies is shown in figure (1). The figure illustrates the variation of the rain attenuation based on the rainfall rate. It can be seen that the attenuation of the rain $(\mathrm{dB} / \mathrm{km})$ is exponentially increase with the increase of rain intensity affects the signal. 


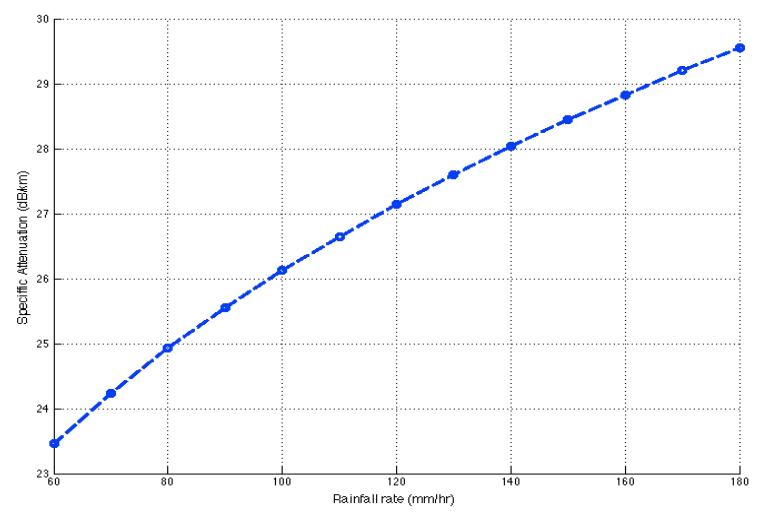

Figure2. Specific attenuation Vs. Rainfall Rate

The relationship between the raindrop size versus the specific attenuation $(\mathrm{dB} / \mathrm{km})$ is shown in figure (2). The rain attenuation increases directly with the drop radius, because the scattering of the higher microwave signals is that the size of raindrops is close to the wavelength, thus the raindrop cross-section will increase in accordance to the raindrop radius.

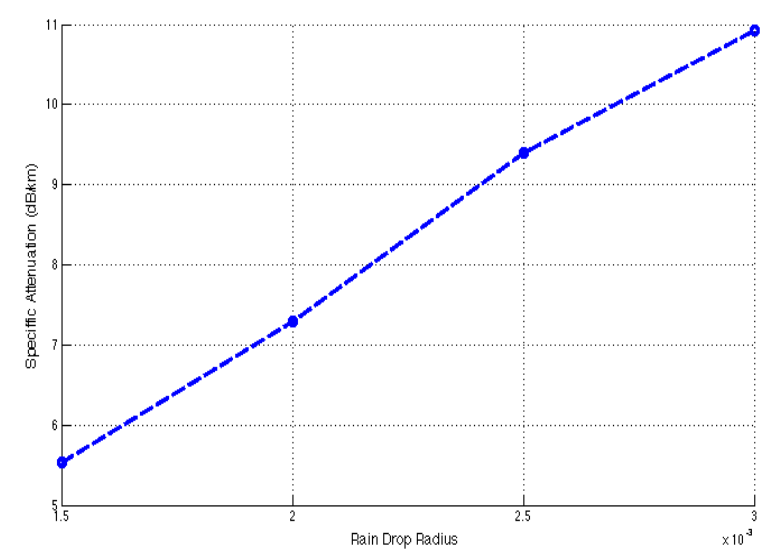

Figure 3. Specific attenuation Vs. Raindrop radius

The rain attenuation of a radio wave propagating in the rain volume of extent $\mathrm{d}$ in the direction of the wave propagation. The rain cell size playing an important role to the rain attenuation, the relationship is plotted in Figure (3), which demonstrates the expected linear relationship between these two quantities.

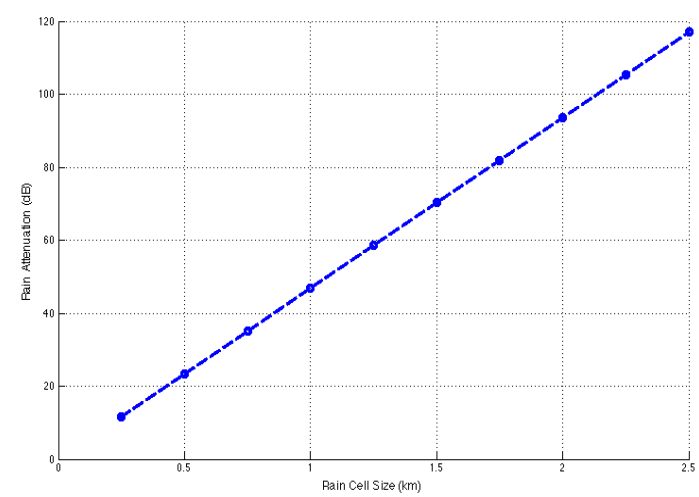

Figure 4.Rain attenuation Vs. Rain cell size

The rain attenuation is related directly to the size of the rain cell where it varies from 0.25 to $2.5 \mathrm{~km}$. This variation of cell size causes more rain attenuation in the signal. Thus, the affected signal path becomes longer, doubling the attenuation each time the cell size increases. 


\section{Discussion And Conclusion}

Rain attenuation is the primary source of signal impairment for HAPS communication systems operating at any frequency above $10 \mathrm{GHz}$. Rain attenuation is caused by absorption and scattering of the signal, in which the raindrop acts as poor dielectric. When the electromagnetic waves propagate through a rainy medium, it will interact with and rain particles causing the scattering. Each particle scatters the energy from the incident signal and reradiates that energy to all directions, where the amount of the signal power will be scattered is dependence on the raindrop size. The analysis has been done in this paper based on the knowledge of rain microstructure parameters such as the raindrop size, rainfall rate and the raindrops size distribution. The drop size distribution was determined by using Palmar-Marshal model based on three parameters: $\mathrm{N}_{0}, \Lambda, \mathrm{R}$ and D.

The analysis shows that the rain attenuation increases in proportion to the increase of the raindrop size, rainfall rate and the rain cell size. The predicted attenuation of $47 \mathrm{~dB}$ has been found for a $1 \mathrm{~km}$ diameter rain cell size.

The increase of the attenuation takes place in accordance to the rain cell size showing the impact of the bigger rain cell produces the highest attenuation amount.

\section{References}

[1]. Ahmad F.M.Z., AssadeqAbolhaoshat Mansour Albendag, "Improving ITU-R Rain Attenuation Model for HAPS Earth-Space Link”, Space Science and Communication (IconSpace), 2013 IEEE International Conference, pp. 56-59.

[2]. Hao, X., Rappaport, T.S., Boyle, R. j., \&Schaffner, j. H. (2000)."Measurement and models for 38-GHz point-to-multipoint radiowave propagation". IEEE journal on selected areas in communications, 18(3), 310-320.

[3]. Zhenwei, Z.,Leke, L.,\&Yumei, L. (2003). “A prediction model of rain attenuation along earth-space path".In IEEE proceedings on antennas, propagation and EM theory (pp. 516-519), November 2003.

[4]. International Telecommunication Union, "Propagation data and prediction methods required for the design of Earth-space telecommunication systems," ITU, Geneva, Switzerl

[5]. Matthew N.O. Sadiku, Numerical Techniques in Electromagnetics, 2nd Edition, CRC Press, 2000.

[6]. Radio meteorological data, CCIR (1981a), report 563-1 (MOD F), Doc. 5/5049-E.

[7]. Marshall, J. S., and Palmer, W.M.K, 1948, "The distribution of raindrops with size," J. Meteor. 5: 165-66.

[8]. Testud, J., S. Oury, R. A. Black, P. Amayenc, and D. Xiankang, 2001: The concept of "normalized" distribution to describe raindrop spectra: a tool for cloud physics and cloud remote sensing. J. Appl. Meteorol., 40, 1118-1140.

[9]. Matthew N.O. Sadiku, Elements of Electromagnetics, 4th Edition, Oxford University Press, 2007.

[10]. H. C. Van De Hulst, Light Scattering by Small Particles. New York. Wiley 1957. 